\title{
Polinomios de Zernike y su aplicación en Oftalmología
}

\author{
MARLON RECARTE ${ }^{1}$ \\ ${ }^{1}$ Universidad Nacional Autónoma de Honduras en el Valle de Sula, mail: marlon.recarte@unah.edu.hn
}

Recibido: 28 de febrero del 2017 / Aceptado: 30 de abril del 2017

\begin{abstract}
Resumen
Any device used for vision needs to be well calibrated, either lenses or the most complex telescope. This requires a good focus of the lenses of the device in question, this will allow us to have an optimal view in the case of lenses or of seeing beyond as the case of a telescope. From 2000, the Zernike polynomials are adopted as the standard in ophthalmological optics. These polynomials are used today in aberrometers, which are devices that measure the aberration of the ocular wavefront or, in other words, are responsible for detecting vision problems we have.
\end{abstract}

Keywords: Ophthalmology, Zernike, Orthogonal polynomials

Cualquier aparato que se utilice para la visión necesita estar bien calibrado, ya sean lentes o el telescopio más complejo. Para ello es necesario un buen enfoque de las lentes que utilice el aparato en cuestión, ya que esto nos permitirá tener una visión óptima en el caso de las lentes o de poder ver más lejos en el caso del telescopio. A partir del año 2000, los polinomios de Zernike se adoptan como el estándar en óptica oftalmológica. Estos polinomios se usan hoy en día en los aberrómetros, que son los aparatos que miden la aberración del frente de onda ocular o, en otras palabras, se encargan de detectar los problemas de visión que tenemos.

Palabras clave: Oftalmología, Zernike, Polinomios ortogonales

\section{Polinomios de Zernike}

$\mathrm{L}$ OS polinomios de ZeRniKe son un conjunto de polinomios ortogonales sobre el disco unidad en dimensión $2, B^{2}=\left\{(x, y) \in \mathbb{R}^{2}: x^{2}+y^{2} \leq 1\right\}$. Su nombre se debe al físico holandés Frits ZeRnike (18881966) ganador del premio Nobel de Física en 1953 por la invención del microscopio de contraste de fase. Dichos polinomios han sido usados en óptica, aberraciones del ojo humano, modelado superficial de córnea [3, 10. Durante las últimas décadas, este conjunto de polinomios se ha convertido en una herramienta universal para cada problema en óptica y oftalmología.

Los polinomios de ZeRnIKE con doble índice son definidos como

$$
Z_{n}^{m}(\rho, \theta)=\left\{\begin{array}{l}
N_{n}^{m} R_{n}^{|m|}(\rho) \cos (m \theta), m \geq 0 \\
N_{n}^{m} R_{n}^{|m|}(\rho) \sin (|m| \theta), m<0
\end{array}\right.
$$

donde $0 \leq \rho \leq 1$ y $0 \leq \theta \leq 2 \pi$ (sin dimensiones) son las coordenadas polares y el doble índice $(n, m)$ con las restricciones: $n \geq 0,|m| \leq n$ y $n-m$ un número par.

La parte radial $R_{n}^{|m|}(\rho)$ es un polinomio de Jacobi reescalado

$$
R_{n}^{|m|}(\rho)=(-1)^{(n-m) / 2} \rho^{m} P_{(n-m) / 2}^{(m, 0)}\left(1-2 \rho^{2}\right)
$$

y son dados de forma explícita por la fórmula:

$$
R_{n}^{|m|}(\rho)=\sum_{s=0}^{\frac{n-|m|}{2}} \frac{(-1)^{2}(n-s) ! \rho^{n-2 s}}{s ! \frac{n+|m|}{2-s} ! s ! \frac{n-|m|}{2-s} !}
$$

El término de normalización $N_{n}^{m}$ es a veces tomado como 1 por simplicidad, dando así sólo ortogonalidad pero no ortonormalidad. Alternativamente, el valor de esta constante de normalización puede definirse como:

$$
N_{n}^{m}=\sqrt{\frac{\left(2-\delta_{0, m}\right)(n+1)}{\pi}}=\sqrt{\frac{2(n+1)}{\pi\left(1+\delta_{0, m}\right)}}
$$

garantizando la ortonormalidad.

Consideraremos la última opción, por lo que la ortonormalidad de los polinomios de ZERNIKE sobre el disco unidad $B^{2}$ en coordenadas polares puede expresarse como:

$$
\int_{0}^{1} \int_{0}^{2 \pi} Z_{n}^{m}(\rho, \theta) Z_{r}^{s}(\rho, \theta) \rho d \rho d \theta=\delta_{n, r} \delta_{m, s}
$$

En la práctica, el uso de un doble índice es a veces engorroso, y existen al menos dos formas diferentes de convertir el doble de índice en un solo índice, conocidos como índice de Noll [9] e índice OSA (donde OSA significa Optical Society of America). En el sistema de Noll, polinomios con términos coseno y seno alternan, comenzando el índice en 1; mientras que en la numeración de la 
OSA, todos los polinomios con el mismo orden radial $n$ son consecutivos y el índice comienza en 0 .

En este trabajo, vamos a utilizar el índice doble $(n, m)$ o el índice OSA $j$ indistintamente. La conversión de un sistema a otro está dado por las expresiones

$$
\begin{aligned}
j & =\frac{n(n+2)+m}{2}, \\
n & =\left\lceil\frac{-3+\sqrt{9+8 j}}{2}\right\rceil, \\
m & =2 j-n(n+2) .
\end{aligned}
$$

Siendo $\lceil\cdot\rceil$ el operador ceiling que da el menor entero que es mayor o igual al argumento.

En el cuadro 1 mostramos las expresiones explícitas

\begin{tabular}{|c|c|c|c|}
\hline$j$ & $n$ & $m$ & Coordenadas Polares \\
\hline 0 & 0 & 0 & 1 \\
\hline 1 & 1 & -1 & $2 \rho \sin (\theta)$ \\
\hline 2 & 1 & 1 & $2 \rho \cos (\theta)$ \\
\hline 3 & 2 & -2 & $\sqrt{6} \rho^{2} \sin (2 \theta)$ \\
\hline 4 & 2 & 0 & $\sqrt{3}\left(2 \rho^{2}-1\right)$ \\
\hline 5 & 2 & 2 & $\sqrt{6} \rho^{2} \cos (2 \theta)$ \\
\hline 6 & 3 & -3 & $\sqrt{8} \rho^{3} \sin (3 \theta)$ \\
\hline 7 & 3 & -1 & $\sqrt{8}\left(3 \rho^{3}-2 \rho\right) \sin (\theta)$ \\
\hline 8 & 3 & 1 & $\sqrt{8}\left(3 \rho^{3}-2 \rho\right) \cos (\theta)$ \\
\hline 9 & 3 & 3 & $\sqrt{8} \rho^{3} \cos (3 \theta)$ \\
\hline 10 & 4 & -4 & $\sqrt{10} \rho^{4} \sin (4 \theta)$ \\
\hline 11 & 4 & -2 & $\sqrt{10}\left(4 \rho^{4}-3 \rho^{2}\right) \sin (2 \theta)$ \\
\hline 12 & 4 & 0 & $\sqrt{5}\left(6 \rho^{4}-6 \rho^{2}+1\right)$ \\
\hline 13 & 4 & 2 & $\sqrt{10}\left(4 \rho^{4}-3 \rho^{2}\right) \cos (2 \theta)$ \\
\hline 14 & 4 & 4 & $\sqrt{10} \rho^{4} \cos (4 \theta)$ \\
\hline
\end{tabular}
para los polinomios de ZERNIKE hasta orden 4 y en la figura 1 se muestran algunos de ellos.

Tabla 1: Polinomios de Zernike

\section{ABERRACIÓN Y FRENTE DE ONDA}

El frente de onda se puede definir como una superficie imaginaria que une todos los puntos en el espacio que son

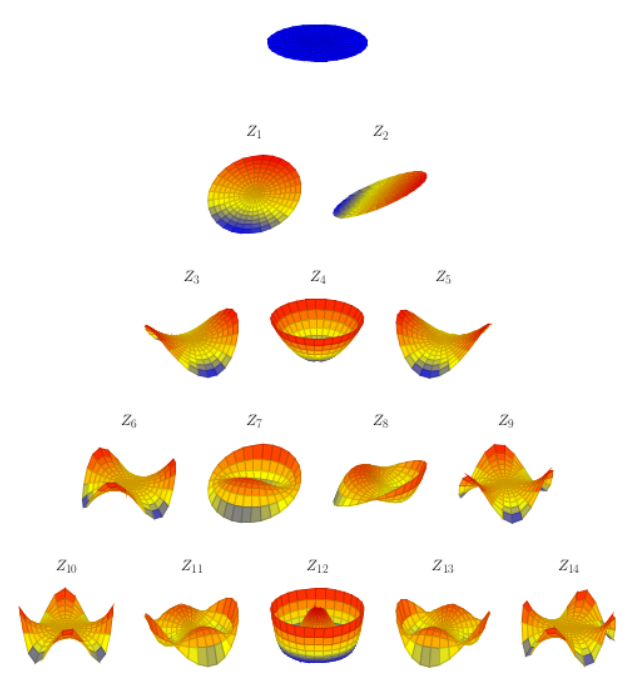

Figura 1: Algunos polinomios de ZeRnike: LA IMAGEN FUe ELABORADA USANDO EL PAQUETE TIKZ DE LATEX

alcanzados en un mismo instante por una onda que se propaga en un medio, es decir aquellos rayos que tienen la misma fase, por lo que un frente de onda es un plano que siempre es perpendicular a todas las ondas que están en fase, ver figura 2

Para un conjunto de vectores paralelos el frente de onda es plano. Para rayos divergentes en un punto o convergentes hacia un punto el frente de onda es esférico. Para los rayos con divergencia o convergencia variado el frente de onda puede tomar cualquiera de las siguientes formas: Elipsoidal, parabólica, las cuales dependen de la naturaleza de la fuente.

Si asumimos que un cuerpo que emite luz lo hace de forma pulsátil, las ondas que emite a la vez siempre están en fase. Por lo tanto, si queremos obtener una buena imagen en la retina de este pequeño punto emisor de luz, tenemos que conseguir que esas ondas en fase converjan en un sólo punto en la retina (o nos aproximaremos lo más que podamos a ese objetivo). El problema es que del punto luminoso salen las ondas en todas direcciones. Es tarea d el ojo hacer converger a las ondas en un lugar de la retina lo más pequeño posible. Cuando el objeto luminoso está lo suficientemente lejos, podemos asumir que los rayos llegan paralelos. Eso significa que el frente de onda es un plano recto. Suponemos que tenemos un ojo perfecto desde el punto de vista óptico. Tras atravesar la primera lente, que es la córnea, los rayos se curvan, por lo que el frente de onda deja de ser un plano recto y se convierte en un plano curvo aunque regular. La forma de ese plano es cóncava si se mira desde el punto de vista de la retina.

Una aberración es cualquier fenómeno que impide que un sistema óptico genere una imagen perfecta. Un ojo se considera aberrado cuando: 


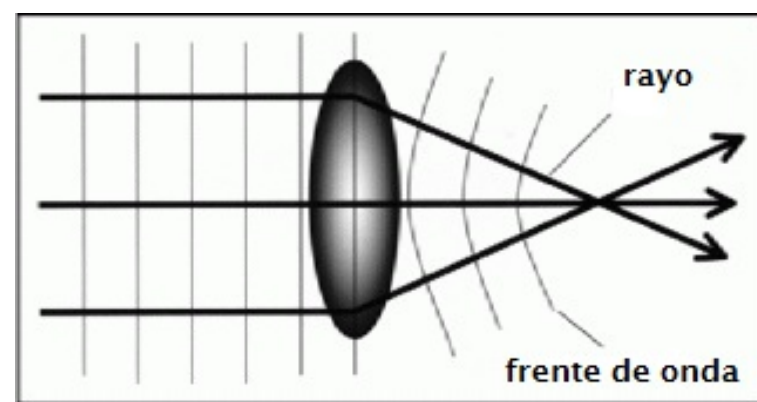

Figura 2: Frente de onda [8]

1. Los rayos no se enfocan en un punto común, retina.

2. La longitud del camino óptico de la trayectoria de un punto del objeto a la imagen no es igual para todos los rayos que pasan a través de la pupila.

3. Los frentes de onda dentro del ojo no son esféricos, por el contrario están distorsionados.

Se quiere estudiar lo que pasa con el ojo real, no con el perfecto. Por tanto sabemos que el frente de onda no va ser perfecto. Pero no podemos entrar dentro del ojo para analizar ese frente de onda por lo que no se estudia el frente de onda que entra en el ojo, sino el que sale. El sistema óptico realmente funciona en las dos direcciones. Si se consigue llegar una luz potente y muy focalizada a un punto de la retina (por defecto se concentra en la retina central), esta luz se refleja, generando un frente de onda de rayos divergentes que se alejan de la retina y se acercan a la córnea. Se forma el mismo frente de onda curvo, que si esta vez lo miramos desde fuera, en vez de cóncavo lo vemos convexo, ver figura 3 Pero tiene que tener exactamente la misma forma que el frente de onda que entra en el ojo.

Generalmente se define un frente de onda esférico como el que se muestra en la figura 4, denominado esfera gaussiana de referencia, que como su nombre lo indica nos sirve de referencia para medir el frente de onda aberrado. Por lo tanto existe un error que se define como la diferencia entre la esfera gaussiana y el frente de onda aberrado.

El frente de onda que sale del ojo es una imagen fidedigna del frente que entra. Por tanto se captura este frente de onda con un aparato. Este aparato se llama aberrómetro. Gracias a un cristal que funciona como un semiespejo se introduce la luz en el ojo. El frente de onda

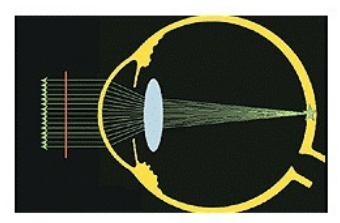

frente de onda plano

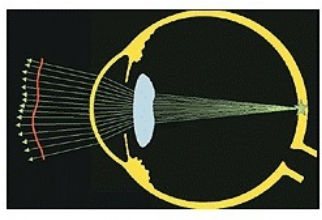

frente de onda deformado
Figura 3: Comparación entre dos frentes de onda[7]

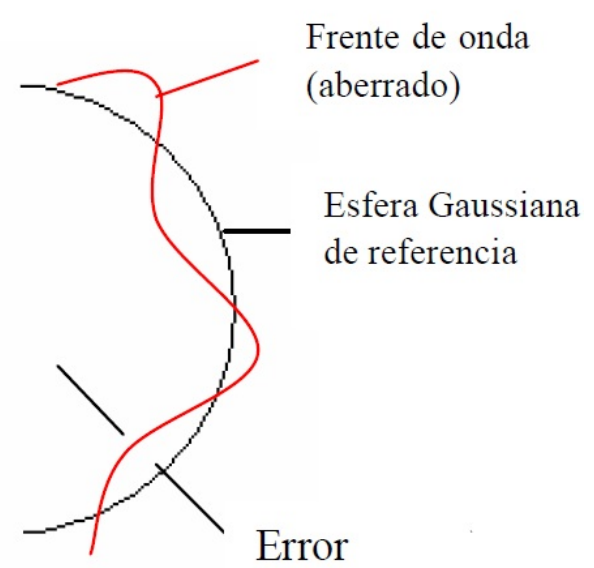

Figura 4: Frente de onda aberrado[7]

queda capturado en la cámara digital del aberrómetro. La imagen es como una rejilla circular de puntos. Si estos puntos están perfectamente alineados se corresponderán con un frente de onda plano y recto. Si algunos puntos, en vez de centrados, están desplazados, entonces el frente de onda presenta irregularidades. La información entonces se traduce en un mapa, en una representación en la que las partes más altas las diferenciamos de las más bajas con un código de colores, ver figura 5.

La Optical Society of America (OSA) recomendó, en los inicios de la interpretación de los mapas de frentes de onda, la adopción de la expansión de polinomios de ZERNIKE como el método estándar para describir el error en el frente de onda de un sistema óptico. Los polinomios de ZERNIKE son considerados como los bloques o funciones básicos de descripción o construcción de cualquier frente de onda, por complejo que éste sea.

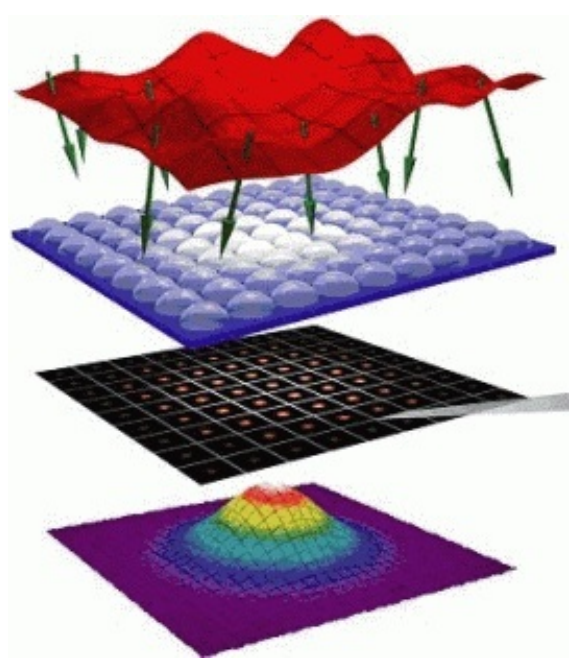

Figura 5: Esquema del aberrómetro[?]

Desde el punto de vista matemático, la forma explícita de la aberración de frente de onda es una función que puede contener muchos términos (en principio infinitos términos); en el sistema de coordenadas cartesiano tiene 
la forma general:

$$
W(x, y)=\sum_{i=0}^{\infty} \sum_{j=0}^{\infty} a_{i j} x^{i} y^{j}
$$

Geométricamente diremos entonces que un polinomio representa una superficie. La forma de esta superficie puede ser simple o compleja, dependiendo del número de monomios que componen el polinomio. A su vez, podemos decir que la superficie que describe el polinomio es la suma de un conjunto de superficies más simples, por ejemplo, planos inclinados, paraboloides, etc.

Otro hecho importante es que diferentes superficies pueden ser generadas por polinomios conformados por el mismo tipo de monomios; lo que hace la diferencia aquí es el valor de los coeficientes en cada caso. Por esta razón, es común describir la superficie simplemente por sus coeficientes (ordenados en algún tipo de histograma) en lugar de escribir la ecuación. Es decir que la aberración del frente de onda $W(\rho, \theta)$ viene dada por un conjunto discreto de datos (mediciones) que se realizan en la pupila.

$$
\left\{x_{i}, y_{i}, W\left(x_{i}, y_{i}\right): i=1,2, \ldots, M\right\}
$$

Se utiliza entonces una aproximación por mínimos cuadrados para reproducir la aberración del frente de onda

$$
W(\rho, \theta) \sim \sum_{j=0}^{N} \lambda_{j} Z_{j}(\rho, \theta)
$$

donde $\lambda_{0}, \lambda_{1}, \ldots, \lambda_{N}$ son las soluciones del sistema de ecuaciones

$$
\left(\begin{array}{cccc}
\left\langle Z_{0}, Z_{0}\right\rangle & \left\langle Z_{1}, Z_{0}\right\rangle & \ldots & \left\langle Z_{N}, Z_{0}\right\rangle \\
\left\langle Z_{0}, Z_{1}\right\rangle & \left\langle Z_{1}, Z_{1}\right\rangle & \ldots & \left\langle Z_{N}, Z_{1}\right\rangle \\
\vdots & \vdots & \ddots & \vdots \\
\left\langle Z_{0}, Z_{N}\right\rangle & \left\langle Z_{1}, Z_{N}\right\rangle & \ldots & \left\langle Z_{N}, Z_{N}\right\rangle
\end{array}\right)\left(\begin{array}{c}
\lambda_{0} \\
\lambda_{1} \\
\vdots \\
\lambda_{N}
\end{array}\right)=\left(\begin{array}{c}
\left\langle W, Z_{0}\right\rangle \\
\left\langle W, Z_{1}\right\rangle \\
\vdots \\
\left\langle W, Z_{N}\right\rangle
\end{array}\right)
$$

Usando el producto escalar discreto

$$
\langle f, g\rangle:=\sum_{i=1}^{M} f\left(x_{i}, y_{i}\right) g\left(x_{i}, y_{i}\right)
$$

Observemos que este producto escalar discreto estará bien definido si el número de medidas $M$ es mayor que el número de elementos de la base de ZERNIKE que se usen, $\mathrm{N}$, y además que estas medidas esten dispuestas en forma adecuada.

En la actualidad, el sensor de Shack-Hartmann utiliza datos sobre una rejilla cartesiana (cuadrada) y la máquina devuelve los coeficientes de cada uno de los polinomios de ZERNIKE resolviendo el sistema de ecuaciones utilizando el método iterativo de Gauss-Seidel.

Los resultados se suelen presentar en un gráfico como el que se muestra en la figura 6, que fue tomada de [5].
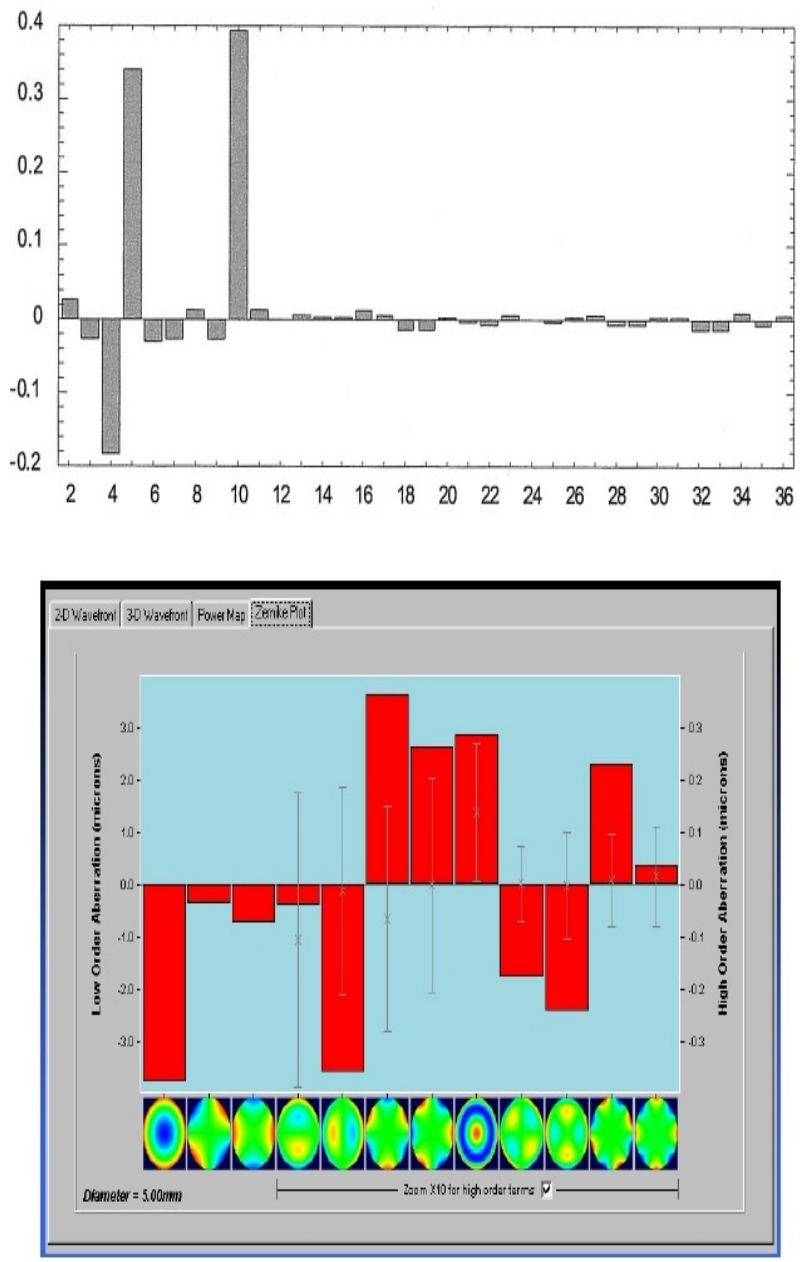

Figura 6: Tabulación de aberraciones [5]

Este sensor envía rayos de luz a través de la retina y hasta que llegan al punto donde convergen todos los rayos de luz que pasan por ésta, entonces estos rayos se reflejan hasta llegar al frente de onda, una vez allí, se calcula la pendiente de la diferencia que hay entre la posición a la que habría llegado ese rayo de luz en el caso de tener un ojo perfecto y la posición a la que ha llegado en realidad.

Por lo tanto, a la hora de trabajar, no se trabaja con la altura de la retina, ya que al ser ésta muy pequeña es una labor muy difícil, sino que se trabaja con pendientes de rectas, de tal modo que si el ojo es un ojo perfecto, todos estas pendientes valdrán cero.

Consecuencia de que los datos que obtenemos son de tipo discreto es que los resultados que obtengamos dependerán de las mediciones que hayamos tomado. De esta forma, si tomamos la misma cantidad de mediciones pero las tomamos en diferentes sitios, obtendremos resultados diferentes.

\section{CONCLUSIONES}

Los polinomios de ZERNIKE constituyen una base matemática adecuada para representar las aberraciones ocu- 
lares. Cada término de ZERNIKE representa la aberración de frente de onda de una de las aberraciones ópticas, es posible probar que su varianza con respecto a una superficie de referencia adecuada es la mínima y, además, el promedio de las distorsiones del frente de onda con respecto a la superficie de referencia es cero.

\section{REFERENCIAS}

[1] ABRAMOWITZ, M. y STEGUN, I. (1970). Handbook of Mathematical functions, 9th. ed. New York: Dover Publ.

[2] ARFKEN, G. y WEBER, H. (2005). Mathematical Methods for Physicists, 6th edition. New York: Elsevier Academic Press, Cambridge University Press.

[3] BORN, M. y WOLF, E. (1975). Principles of Optics, 5th ed. New York: Pergamon.

[4] HOWLAND, C. (2000). The history and methods of ophthalmic wavefront sensing. J Refract Surg. Vol. 16, S552-S553.

[5] L.THIBOS; APPLEGATE, R.; SCHWIEGERLIN, J. y WEBB, R. (2000). Standards for reporting the optical aberrations of eyes. Vision Science and its Applications, SuC1.

[6] MAHAJAN, V. (1994). Zernike circle polynomials and optical aberrations of systems with circular pupils. Applied Optics Vol. 33, 8121-8124.

[7] MALACARA, D. (EIBPOA 2015), Polinomios de Zernike para representar la aberración de un frente de onda. URL http://paginas .matem. unam.mx/ eibpoa2015/images/Malacara.pdf

[8] MEJÍA, Y. (2011). El frente de onda y su representación con polinomios de Zernike. Cien. tecnol. salud. vis. ocul, 9, No.2, 145-166.

[9] NOLL, R. (1976). Zernike polynomials and atmospheric turbulence. J. Opt. Soc. Am. 66, 207-211.

[10] RAMOS, D. (2014). Mathamatical modelling in biomedical optics and ophtal-mology. Tesis, Universidad de Almería.

[11] RAMOS, D.; SÁNCHEZ, M. y FERNÁNDEZ, M. (2015). Optimal sampling patterns for zernike polynomials, (preprint). Preprint submitted to Applied Mathematics and Computation.

[12] THIBOS, L. (2001). Wavefront data reporting and terminology. J Refract Surg. Vol. 17, S578-S583.

[13] VIDAL, R. (2011). Entendiendo e interpretando las aberraciones ópticas. Cien. tecnol. salud. vis. ocul, 9 , No.2, 105-122. 